their telescopes. Two new items of equipment, likely to come into operation soon, one at the Department of Astrophysies at Oxford University, and the other at the Royal Observatory, Edinburgh, should help astronomers make the most of their measurements. The Oxford equipment is a furnace to simulate conditions in the solar atmosphere, and will help in the interpretation of solar spectra; the Edinburgh instrument, called grandly the "Galaxy" machine, is to measure the brightness and position of stars from photographic plates.

The furnace now being built at the Department of Astrophysies at Oxford University, which makes a speciality of solar research, should be working early next year, when the first task will be to observe absorption lines of iron. The furnace consists of a carbon tube about 4 feet long, with an internal diameter of 2 inches, making it, the Oxford astronomers believe, the largest furnace to be used for this kind of work. The tube is heated by an a.c. current passing through it, and is supported in a vacuum. The sample inside the tube vaporizes, and the absorption lines are seen when light from a continuum source is passed. through the gas. It is hoped that the power input to the furnace of $200 \mathrm{~kW}$ will raise the temperature inside the tube to $2,500^{\circ} \mathrm{C}$, which should be high enough to give information useful in working out the abundance of iron in the Sun.

At the Royal Observatory, Edinburgh, final tests of the Galaxy plate measuring machine are in progress, and it is expected to be working in the autumn. In its work on the formation and evolution of stars, the observatory uses a design of telescope known as the Schmidt, which is able to photograph large fields of view, so that many thousands of stars may be recorded in a single exposure. Hitherto a hand operated machine has been used to measure the brightness and position of individual stars from the plates. The Galaxy machine, designed by Dr V. C. Reddish of the Royal Observatory, Edinburgh, and Professor P. B. Fellgett of the University of Reading, together with Ferranti, does this automatically.

The Galaxy machine works in two phases. A flying spot scans all the star images on the photographic plate, and the machine prints out the star positions on computer tape to an accuracy of $16 \mu$. At this stage the machine distinguishes between stars and faults in the photographic plate. The output tape from this part of the operation is then used as the input tape for the next phase, and is fed back into the machine. A spiral scan finds the stars listed and compares them with a catalogue in the machine's memory. In this process the brightness is found to within about 2 per cent and the position to about one micron.

The tedious process of plate analysis will be greatly speeded up by the Galaxy machine. It will deal with 1,000 stars an hour, compared with Edinburgh's manually operated machine which measures the brightness of 200 stars per hour, but the positions of only 50 or 60 per hour. One of the applications will be to find the colour temperature of stars by measuring the brightnesses on plates taken in light of different wavelengths. Because the machine will normally be fed with a plate and left to run on its own, it will examine every star on the plate and so at first will be used for a statistical analysis rather than for the examination of particular stars.
As it is now, the Galaxy machine is unable to distinguish between different kinds of star, and indeed is unable to tell a star from a galaxy. A development of the machine which will make these distinctions by examining the shapes of the images is already being discussed at Edinburgh.

\section{No More Smallpox}

LAsT year the World Health Organization began what it hopes will be an intensive campaign to eradicate smallpox from the world during the next decade. The WHO asked for voluntary contributions from all member states, and two-thirds of the countries planned to start or intensify national programmes by late last year. The rest aimed to begin this year. In an attempt to decide the best strategy for the campaign, WHO organized a meeting of its Scientific Group on the Eradication of Smallpox last October, and the group's findings have just been published.

On the face of things, complete eradication is well within the bounds of possibility. Europe, North America, Australia and Oceania have achieved eradication and the biology of the virus is simple. There is no carrier state, infections that do not lead to overt symptoms are rare and there is no animal reservoir of the disease. The only unfavourable characteristic is the ease with which the virus spreads. Eradication is only meaningful when smallpox has been eliminated from entire continents.

Eradication on a world scale is simply a matter of logistics - the organization of coordinated international vaccination programmes, which, of course, demand an adequate supply of effective vaccine, and the provision of rigorous surveillance. But as past experiences in several countries prove, eradication programmes repeatedly break down through lack of supervision. Either because they are overworked or disinclined to suffer the inconveniences of field work, supervisors make false reports; it is not unknown, for example, for the number of vaccinations reported to exceed by far the total population. The tendency of populations to conceal cases does not help and, in the past, vaccination campaigns have failed because the supply of vaccine has run out. Only freeze-dried vaccine is stable in the tropics.

In the light of these experiences the WHO has called for extra funds to establish regional and international reference laboratories to provide training and screening facilities and also laboratories for the production of freeze-dried vaccine. Unless production of vaccine is increased, supplies are likely to remain insufficient for years to come.

\section{School Reforms}

THE Schools Council, set up in 1964, has now published an account of its activities during its first three years (HMSO, $3 s .6 d$.). The council's first function was to find ways of reforming the school curriculum in England and Wales, but it is also responsible for coordinating and reforming school exams, and has initiated curriculum development in several new areas of work. Part of its work, involving progress in mathematics, science and modern languages, is being undertaken with the cooperation of the Nuffield Fourdation.

The council has an annual budget of about $\mathfrak{£} 1 \cdot 25$ 